\title{
Spatial probability learning by experimentally naive cats and monkeys
}

\author{
J. M. WARREN \\ Pennsylvania State University, University Park, Pennsylvania 16802
}

\begin{abstract}
A group of 18 cats and eight monkeys learned a 70:30 spatial probability problem in the WGTA, and 16 cats were trained in the Grice box. Half the subjects in each group were pretrained on 15 spatial reversals before testing on the probability learning task. The remaining animals received no prior training. Reversal pretraining did not facilitate or retard probability learning in any set of subjects. All subjects learned to maximize. Although the cats tested in the Grice box required significantly more training than those trained in the WGTA, the performance of the two groups of cats and the monkeys was qualitatively similar. The performance of naive cats appears to be essentially the same as that of other naive mammals.
\end{abstract}

In probability learning, rats and monkeys maximize or match systematically (Bitterman, 1965, 1975), but cats with extensive learning set experience do not (Poland \& Warren, 1967, 1968; Warren \& Beck, 1966). Experimentally naive cats, however, quickly learn consistently to select the more frequently rewarded alternative in probability learning on visual cues (Schweikert \& Treichler, 1969).

The discrepant results of experiments with naive and highly sophisticated cats suggest that prior training may affect cats' performance on probability learning tasks. This hypothesis was tested by comparing the performance on a 70:30 spatial probability learning problem of animals that had or had not previously been trained on 15 reversals of a spatial discrimination habit. The spatial reversals were learned with consistent reinforcement, so, depending on one's theoretical predilections, two contradictory predictions might be made regarding transfer from reversal to probability learning. Spatial reversal training might enhance the salience of spatial cues and thereby facilitate spatial probability learning (Sutherland \& Mackintosh, 1971). Alternatively, consistent reinforcement during reversal training might engender a "win-stay lose-shift" hypothesis (Levine, $1965)$ that would retard development of the "win-orlose/stay" rule necessary for maximizing in probability learning.

Two different groups of cats were tested in apparatus requiring manipulatory (WGTA) or locomotor (Grice box) responses to see if situational factors affect probability learning by cats. In addition, a group of monkeys were tested in the WGTA to provide a direct comparison between species tested under highly similar conditions in probability learning.

\section{METHOD}

\section{Subjects}

Eighteen of 32 experimentally naive adult mongrel cats were tested in the WGTA, and 14 were tested in the Grice box; half the animals in each apparatus were tested on probability learning with no prior training, and half were tested after reversal training. The groups of cats were balanced for litter membership by the split-litter technique.

The eight adolescent rhesus monkeys (Macaca mulatta) studied were also experimentally naive, and they were randomly assigned to two groups of four that were tested on probability learning after reversal training or no pretraining.

All of the subjects were given sufficient adaptation training with nondifferential reinforcement to insure prompt responses before testing on the learning problems. The animals were tested under 18-24 $\mathrm{h}$ of food deprivation. The cats were reinforced for correct responses with tuna or pork kidney, and the monkeys were rewarded with peanuts or raisins.

\section{Apparatus and Stimuli}

The objects used in the WGTA (Harlow, 1949) were identical black $7.6-\mathrm{cm}$ squares, presented over the right and left foodwells of a gray tray. The vertical plaques presented in the Grice box for cats (Warren, 1975) were black 10.2-cm squares.

\section{Procedure}

Reversal learning. The pretrained cats and monkeys first learned to choose their initially nonpreferred position and were then trained on 15 reversals of the discrimination habit to a criterion of 18 correct in one session of 20 noncorrection trials.

Probability learning. Neither position was reinforced on the first trial of the first session. The position that was not chosen on this preference trial was designated as the more frequently rewarded alternative and was reinforced on $70 \%$ of the subsequent trials in an irregular sequence. The opposite position, the less frequently rewarded alternative, was rewarded on the remaining $30 \%$ of the trials. The criterion was 18 responses to the more frequently rewarded alternative in 20 noncorrection trials for 5 consecutive days.

\section{RESULTS}

\section{Reversal Learning}

The pretrained cats and monkeys learned successive reversals with progressively greater efficiency. The monkeys made only $9 \%$ as many errors on Reversal 15 as on Reversal 1. The corresponding values for the cats trained in the WGTA and the Grice box were $45 \%$ and $17 \%$, respectively. 
Table 1

Mean Trials to Criterion in Probability Learning

\begin{tabular}{llrrrrr}
\hline & & \multicolumn{4}{c}{ Treatment } \\
\cline { 3 - 4 } \cline { 6 - 7 } \cline { 5 - 6 } \cline { 5 - 6 } Species & Apparatus & Mean & SD & & Mean & SD \\
\cline { 3 - 4 } Cats & Grice Box & 194 & 129 & & 403 & 294 \\
Cats & WGTA & 120 & 87 & & 142 & 152 \\
Monkeys & WGTA & 90 & 53 & & 205 & 111 \\
\hline
\end{tabular}

\section{Probability Learning}

The means and the standard deviations of trials to criterion on the probability learning problem are given in Table 1. In each set of subjects, the animals that had been pretrained on reversals made fewer errors than did those tested on probability learning without previous training, but $t$ tests indicate that none of the differences was statistically significant.

The scores of the two groups of cats trained in the WGTA and the Grice box were subjected to a 2 by 2 factorial analysis of variance, with apparatus and training the main effects. The analysis revealed that apparatus was a significant source of variation $[F(1,27)=6.90$, $\mathrm{p}<.05]$. Neither training nor the Apparatus by Training interaction was significant.

The data for the cats tested in the WGTA and the monkeys were similarly analyzed, with species and treatment the main effects. Neither of these factors was significant, nor was their interaction.

\section{DISCUSSION}

All of the subjects solved the probability learning problem with reasonable facility, the totally inexperienced animals as well as those that had learned 15 reversals of a spatial discrimination habit. There is thus no evidence that modest amounts of previous training with spatial cues and consistent reinforcement either facilitates or retards probability learning on spatial cues. These findings confirm the observation of rapid visual probability learning by naive cats (Schweikert \& Treichler, 1969), but they leave obscure the reasons why highly sophisticated cats do not consistently maximize or match systematically in probability learning (Poland \& Warren, 1967, 1968; Warren \& Beck, 1966).

Cats trained in the WGTA require significantly fewer trials to master a probability learning task than do cats trained in a Grice box. It is reassuring, however, that the groups observed in these different experimental situations behaved in a qualitatively similar fashion. Reversal pretraining was ineffective for the animals in both apparatuses, and all of the subjects in both groups eventually developed a high level of maximizing behavior.

The results obtained from the monkeys and cats were examined for evidence of any peculiarities in probability learning by cats. None was found. The mean number of test sessions preceding criterion was almost identical, 5.5 for eight monkeys, and 4.5 for 18 cats. Sequential analyses of responses on successive trials also failed to reveal any substantial differences between species. It seems most likely that the behavior of naive cats is essentially the same as that of other mammals in probability learning.

\section{REFERENCES}

Bitterman, M. E. Phyletic differences in learning. American Psychologist, 1965, 20, 396-410.

Bitte rman, M. E. The comparative analysis of learning. Science, 1975, 188, 699-709.

Harlow, H. F. The formation of learning sets. Psychological Review, 1949, 56, 51-65.

Levine, M. Hypothesis behavior. In A. Schrier, H. F. Harlow, \& F. Stollnitz (F.ds.), Behavior of nonhuman primates (Vol. 1). New York: Academic Press, 1965.

Poland, S. F., \& Warren, J. M. Spatial probability learning by cats. Psychonomic Science, 1967, 8, 487-488.

Poland, S. F., \& Warren, J. M. Cats' performance on visual probability learning problems after prolonged training. Journal of Comparative and Physiological Psychology, 1968, 65, 545-546.

Schweikert, G. E., \& Treichler, F. R. Visual probability learning and reversal in the cat. Journal of Comparative and Physiological Psychology, 1969, 67, 269-272.

Sutherland, N. S., \& Mackintosh, N. J. Mechanisms of animal discrimination. New York: Academic Press, 1971.

WARren, J. M. Overtraining, extinction and reversal learning by kittens. Animal Learning \& Behavior, 1975, 3, 340-342.

WARREN, J. M., \& Bеск, C. H. M. Visual probability learning by cats. Journal of Comparative and Physiological Psychology, $1966,61,316-318$.

(Received for publication May 8, 1980.) 